\section{Tendências e desigualdades na insegurança alimentar durante a pandemia de COVID-19: resultados de quatro inquéritos epidemiológicos seriados}

\section{Trends and inequalities in food insecurity during the COVID-19 pandemic: results of four serial epidemiological surveys}

\section{Tendencias y desigualdades en la inseguridad alimentaria durante la pandemia de COVID-19: resultados de cuatro encuestas epidemiológicas seriadas}

\author{
Leonardo Pozza dos Santos 1 \\ Antônio Augusto Schäfer 2 \\ Fernanda de Oliveira Meller ${ }^{2}$ \\ Jenifer Harter ${ }^{3}$ \\ Bruno Pereira Nunes 4 \\ Inácio Crochemore Mohnsam da Silva 4 \\ Debora da Cruz Payão Pellegrini 3
}

doi: $10.1590 / 0102-311 \times 00268520$

\section{Resumo}

O objetivo foi analisar tendências e desigualdades na prevalência de insegurança alimentar na pandemia de COVID-19, de acordo com fatores sociodemográficos e com medidas de distanciamento social. Dados de quatro inquéritos epidemiológicos seriados sobre a COVID-19 desenvolvidos entre maio e junho de 2020, com adultos e idosos residentes na cidade de Bagé, Rio Grande do Sul, Brasil. Insegurança alimentar foi avaliada por meio da versão curta da Escala Brasileira de Insegurança Alimentar (EBIA), com o período recordatório adaptado ao início das medidas de distanciamento social no município. As características sociodemográficas e a adoção de medidas de distanciamento social foram analisadas, e suas associações com a insegurança alimentar foram avaliadas utilizando-se o teste de qui-quadrado. A tendência temporal da insegurança alimentar de acordo com tais características foi avaliada usando-se regressão linear. As desigualdades na insegurança alimentar foram avaliadas utilizando-se o indice angular de desigualdade e o indice de concentração. Dos 1.550 indivíduos estudados, 29,4\% (IC95\%: 25,0; $34,4)$ apresentaram insegurança alimentar. A análise de desigualdade mostrou maior concentração da insegurança alimentar entre os mais jovens, os menos escolarizados e os que residiam em domicílios com cinco moradores ou mais. Ao longo dos quatro inquéritos, a prevalência de insegurança alimentar reduziu mais acentuadamente entre os mais jovens, naqueles que residiam em domicílios com até dois moradores e com dois ou mais trabalhadores. Evidenciou-se forte associação da insegurança alimentar com os aspectos sociodemográficos dos entrevistados, o que pode indicar o potencial impacto econômico da pandemia na situação alimentar dos domicílios.

Segurança Alimentar e Nutricional; COVID-19; Epidemiologia Nutricional

\author{
Correspondência \\ L. P. Santos \\ Curso de Nutrição, Universidade Federal do Pampa. \\ Rua Luiz Joaquim de Sá Britto s/n, Itaqui, RS \\ 97650-000, Brasil. \\ leonardo_pozza@yahoo.com.br \\ 1 Universidade Federal do Pampa, Itaqui, Brasil. \\ 2 Programa de Pós-graduação em Saúde Coletiva, Universidade \\ do Extremo Sul Catarinense, Criciúma, Brasil. \\ 3 Universidade Federal do Pampa, Uruguaiana, Brasil. \\ 4 Universidade Federal de Pelotas, Pelotas, Brasil.
}




\section{Introdução}

Desde fevereiro de 2020, quando o primeiro caso de COVID-19 foi confirmado no Brasil, até o dia 8 de dezembro do mesmo ano, o número de infectados no país superou a marca de 6 milhões e 600 mil indivíduos, chegando a quase 180 mil óbitos (https://covid.saude.gov.br/, acessado em 08/Dez/2020). Especificamente no Estado do Rio Grande do Sul, até 8 de dezembro de 2020 havia mais de 350 mil casos de COVID-19 com mais de 7 mil mortes (http://ti.saude.rs.gov.br/covid19/, acessado em 08/ Dez/2020).

Entre as diversas ações empregadas para frear a propagação do vírus no Brasil, medidas de distanciamento social foram adotadas, o que levou a uma diminuição da atividade econômica com consequente queda do Produto Interno Bruto (PIB) 1 e aumento do desemprego 2 no primeiro trimestre de 2020. Tais fatores podem ter um efeito importante na garantia do Direito Humano à Alimentação Adequada (DHAA) 3,4, ocasionando aumento na prevalência de insegurança alimentar domiciliar 5 . De modo a minimizar tais impactos, o governo sancionou a Lei no 13.982/2020 6 que, entre outras coisas, estabeleceu medidas excepcionais de proteção social, com o pagamento de um auxílio emergencial para as populações em vulnerabilidade social.

Entende-se por insegurança alimentar a falta de acesso regular a alimentos de qualidade e em quantidade suficiente, ou quando tal acesso compromete outras necessidades essenciais de uma família 7. No Brasil, houve um declínio na prevalência de insegurança alimentar entre os anos de 2004 e 2013, de acordo com a Pesquisa Nacional de Amostra por Domicílios (PNAD) 8,9,10, embora em 2013 esta condição ainda atingisse quase um quarto dos domicílios brasileiros (22,9\%) 9,10. Estimativas mais recentes têm evidenciado, contudo, um aumento na prevalência de insegurança alimentar a partir de 2015, quando iniciou um intenso processo de crise econômica e política no país 11,12. Dados da Pesquisa de Orçamentos Familiares (POF), divulgados recentemente pelo Instituto Brasileiro de Geografia e Estatística (IBGE), mostraram que a prevalência de insegurança atingiu 36,7\% dos domicílios brasileiros em 2017/2018 12. Tal situação pode estar sendo agravada pela pandemia de COVID-19 em 2020.

Relatório do comitê de segurança alimentar da Organização das Nações Unidas para Agricultura e Alimentação (FAO) alerta que o quadro de insegurança alimentar tende, de fato, a se agravar em decorrência da pandemia ${ }^{13}$. É importante destacar que, em contextos de crise, populações mais vulneráveis apresentam maior risco de agravo no quadro de insegurança alimentar 11,14,15,16. Além disso, o relatório global para crises alimentares estima que o número de pessoas vivendo em situação de insegurança alimentar pode dobrar em 2020 na comparação com 2019, por conta da crise econômica ocasionada pela pandemia de COVID-19 5,17.

Em um país com acentuadas desigualdades sociais como o Brasil 18,19, as medidas de distanciamento social adotadas para frear a propagação do novo coronavírus podem agravar ainda mais a insegurança alimentar de determinados subgrupos populacionais 20 , visto que nem todos podem ou têm condições de cumprir tais medidas sem que haja prejuízos econômicos com consequente piora no acesso à alimentação. Nesse sentido, monitorar a prevalência de insegurança alimentar dos domicílios brasileiros em tempos de pandemia, considerando não apenas aspectos socioeconômicos e demográficos, mas também a adoção de medidas de distanciamento social, é de extrema importância a fim de garantir que o DHAA seja assegurado mesmo com as condições adversas que o país enfrenta.

Assim, o presente estudo objetivou descrever as tendências e desigualdades na prevalência de insegurança alimentar durante a pandemia de COVID-19 no Município de Bagé, Rio Grande do Sul, Brasil, de acordo com os fatores sociodemográficos e com as medidas de distanciamento social adotadas pelo município, baseando-se em quatro inquéritos epidemiológicos seriados de base populacional.

\section{Métodos}

Esta pesquisa foi realizada com dados oriundos do projeto intitulado: Inquérito Populacional da COVID-19 em Bagé: Prevalência, Impactos Econômicos e na Saúde Mental da População, desenvolvido pela Universidade Federal do Pampa (Unipampa) em parceria com a Universidade Federal de Pelotas (UFPel) e com a Secretaria de Saúde e Atenção à Pessoa com Deficiência do Município de Bagé. Esse 
projeto teve como objetivo principal estimar a prevalência de pessoas infectadas pelo SARS-CoV-2 na cidade de Bagé, com base em quatro inquéritos epidemiológicos seriados.

Bagé é um município localizado na metade sul do Rio Grande do Sul, mais especificamente na Região da Campanha e, de acordo com estimativa do IBGE, tinha cerca de 120 mil habitantes em 2019. Em termos econômicos, Bagé apresentou PIB per capita de BRL 24.601,29 e Índice de Desenvolvimento Humano (IDH) de 0,740 em 2017. Além disso, em 2010, Bagé apresentou taxa de analfabetismo de 5,1\% e mortalidade infantil de 17,64 óbitos a cada mil nascidos vivos (http://cidades.ibge.gov.br/ brasil/rs/bage/panorama).

Desde o dia 19 de março de 2020, o Município de Bagé tem vivido uma situação de calamidade pública, decretada pelo poder executivo (Decreto Municipal no 050/2020 21), que impõe medidas mais rígidas de distanciamento social, com suspensão das atividades de ensino, fechamento de clubes sociais e esportivos, academias, cinemas e bares. Estabelecimentos comerciais considerados essenciais para o abastecimento da população, como supermercados, farmácias e postos de gasolina, permaneceram abertos com horários específicos de funcionamento e com a adoção de medidas de higiene e distanciamento dos clientes.

O presente estudo usou dados de quatro inquéritos epidemiológicos seriados com delineamento transversal, de base populacional, cuja população-alvo foi composta por indivíduos com 18 anos ou mais de idade, residentes na zona urbana do Município de Bagé. O projeto seguiu delineamento semelhante ao EPICOVID-19 22, isto é, processo de amostragem realizado em múltiplos estágios, com seleção aleatória de 400 indivíduos selecionados por conglomerados em cada um dos quatro inquéritos realizados. A definição dos conglomerados foi feita com base na grade de setores censitários de Bagé que seria utilizada pelo IBGE no Censo Demográfico de 2020. Inicialmente, foram selecionados aleatoriamente 40 setores censitários dos 167 localizados na zona urbana do município. Em uma segunda etapa, foram selecionados 10 domicílios dentro de cada setor, resultando em 400 domicílios visitados em cada inquérito epidemiológico, totalizando 1.600 domicílios visitados no total. Os quatro inquéritos epidemiológicos foram realizados entre os dias 7 e 9 de maio, 22 e 26 de maio, 8 e 10 de junho e entre os dias 23 e 26 de junho de 2020.

A coleta de dados foi realizada por agentes comunitários de saúde e técnicos de enfermagem vinculados à rede de atenção básica do município, que foram previamente treinados para a aplicação dos questionários e para a realização dos testes sorológicos para a averiguação da infecção por SARS-CoV-2. Ao todo, participaram das entrevistas e testes 19 profissionais da atenção básica, que foram autorizados pela Secretaria de Saúde e Atenção à Pessoa com Deficiência do Município de Bagé a aplicar os questionários, utilizando os devidos materiais de proteção individual nas coletas de dados.

Para o presente estudo, o desfecho analisado foi a situação de insegurança alimentar domiciliar, definida baseando-se na versão curta da Escala Brasileira de Insegurança Alimentar (EBIA) proposta por Santos et al. 23. Apesar de não captar os diferentes níveis de intensidade da insegurança alimentar, tal instrumento permite rastrear famílias com insegurança alimentar e foi escolhido devido ao baixo número de questões, haja vista a necessidade de aplicação rápida dos questionários. Para o presente estudo, foi realizada uma adaptação da versão curta da EBIA no que diz respeito ao período recordatório do questionário: em vez do período de três meses, como consta na versão original, optou-se por considerar como período recordatório o início das medidas de distanciamento social (março de 2020). Foram classificados como inseguros aqueles domicílios que apresentaram pelo menos uma resposta positiva nas questões do instrumento utilizado.

Os fatores sociodemográficos analisados neste estudo foram: sexo (masculino/feminino), idade (coletada em anos completos e categorizada em 20-39, 40-59 e $\geq 60$ anos), escolaridade (nenhuma/ Ensino Fundamental, Ensino Médio ou Ensino Superior), cor da pele autorreferida (branca, preta ou parda), número de moradores por domicílio (1-2, 3-4 e $\geq 5$ moradores) e presença de moradores menores de 18 anos no domicílio (não/sim). Optou-se por excluir da análise respondentes menores de 20 anos (47 indivíduos), visto que, na maioria das vezes, não são eles os responsáveis pelas compras de alimentos do domicílio, o que poderia interferir nas respostas sobre insegurança alimentar.

Os fatores relacionados às medidas de distanciamento social analisados no presente estudo foram: número de trabalhadores por domicílio, capacidade de atender as medidas de distanciamento social orientadas pelas autoridades e a rotina de atividades em tempos de pandemia. Para a variável número de trabalhadores por domicílio, foi questionado o total de moradores naquele domicílio e quais 
estavam trabalhando no momento da entrevista, independentemente se o trabalho era presencial ou remoto. Os entrevistados também foram questionados se estavam conseguindo cumprir as medidas de distanciamento social orientadas pelas autoridades por meio da seguinte pergunta: "Com relação ao distanciamento social que está sendo orientado pelas autoridades de saúde, ou seja, ficar em casa e evitar contato com outras pessoas, quanto você acha que está conseguindo fazer?" (variável categorizada em muito pouco ou pouco, mais ou menos ou praticamente isolado). Por fim, os entrevistados foram questionados sobre sua rotina em tempos de pandemia, pela seguinte questão: "Como tem sido a sua rotina de atividades?” (variável categorizada em fica em casa o tempo todo, sai às vezes, sai todos os dias para trabalhar ou outra atividade).

Para testar a associação da insegurança alimentar com as características demográficas e de distanciamento social, utilizou-se o teste de qui-quadrado, considerando-se um nível de 5\% de significância e intervalo de $95 \%$ de confiança (IC95\%). Na análise, foi considerada a amostra total do estudo, bem como os quatro inquéritos epidemiológicos seriados de forma estratificada, com o objetivo de verificar diferenças na prevalência de insegurança alimentar entre o início de maio e o fim de junho de 2020.

A análise de tendência temporal da prevalência de insegurança alimentar entre maio e junho de 2020, de acordo com as características sociodemográficas e com as medidas de distanciamento social analisadas, foi realizada usando-se os modelos de regressão linear, em que o desfecho foi a prevalência de insegurança alimentar em cada um dos subgrupos analisados e a exposição foi a rodada de coleta de dados. A opção pela regressão linear simples para a análise de tendência foi feita pela ausência de autocorrelação serial entre as medidas inseridas no modelo, atestada mediante teste de BreuschGodfrey 24,25,26.

Por fim, análises formais de desigualdades absolutas e relativas na prevalência de insegurança alimentar de acordo com idade, escolaridade e número de moradores no domicílio foram realizadas complementarmente usando-se o índice angular de desigualdade (slope index of inequality - SII) e o índice de concentração (concentration index - CIX). O SII é uma medida de desigualdade absoluta obtida com base na regressão logística entre a prevalência de insegurança alimentar com as características sociodemográficas dos entrevistados; no caso do presente estudo idade, escolaridade e número de moradores no domicílio. Tal abordagem permite calcular a diferença na prevalência de insegurança alimentar entre os grupos extremos da variável de estratificação (mais favorecidos vs. menos favorecidos), levando-se em consideração o tamanho da amostra em cada subgrupo desta variável. Valores positivos indicam que a frequência do desfecho é maior no grupo mais privilegiado, já os valores negativos indicam o oposto $27,28,29$.

Já o CIX utiliza abordagem similar à do coeficiente de Gini e, assim como o SII, leva em consideração todos os subgrupos da variável independente. Na análise do CIX, quanto mais afastados do zero os valores, maior a desigualdade, com valores positivos indicando uma diferença pró-grupo mais privilegiado e valores negativos indicando diferença pró-grupo menos privilegiado 27,28,29. As análises de desigualdade com base no SII e no CIX foram estratificadas por inquérito epidemiológico, com o objetivo de detectar diferenças nas desigualdades absoluta e relativa entre o início de maio e o fim de junho de 2020.

Todas as análises foram realizadas no pacote estatístico Stata, versão 16.1 (https://www.stata. com), e levaram em consideração o efeito de delineamento da pesquisa. O presente estudo foi aprovado pelo Comitê de Ética da Faculdade de Medicina, Universidade Federal de Pelotas (UFPel), sob parecer no 4.078.044.

\section{Resultados}

Dos 1.553 domicílios selecionados para fazer parte do estudo e incluídos na análise, três foram excluídos por apresentarem questionários incompletos. Assim, este estudo incluiu 1.550 adultos (idade igual ou superior a 20 anos) entrevistados entre o início de maio e o fim de junho de 2020, sendo $66 \%$ do sexo feminino (Tabela 1 ). 
Do total de entrevistados, mais de um terço possuía 60 anos ou mais e quase a metade informou não possuir escolaridade ou ter cursado, no máximo, o Ensino Fundamental. Cerca de 80\% se autodeclararam com cor da pele branca, pouco mais de 10\% afirmaram residir em domicílio com cinco ou mais pessoas, 36\% dos domicílios tinham a presença de menores de 18 anos entre os seus residentes e cerca de um terço dos entrevistados afirmou residir em domicílios sem trabalhadores, isto é, com a presença de desempregados e/ou aposentados. Com relação às medidas de distanciamento social, 64\% dos entrevistados afirmaram ter estado praticamente isolados desde que as medidas de distanciamen-

\section{Tabela 1}

Características sociodemográficas e de distanciamento social em indivíduos adultos. Bagé, Rio Grande do Sul,

Brasil $(n=1.550)$

\begin{tabular}{|c|c|c|}
\hline Características sociodemográficas & $\mathbf{n}$ & $\%$ (IC95\%) \\
\hline \multicolumn{3}{|l|}{ Sexo } \\
\hline Masculino & 512 & $34,0(30,8 ; 37 ; 4)$ \\
\hline Feminino & 995 & $66,0(62,6 ; 69,2)$ \\
\hline \multicolumn{3}{|l|}{ Idade (anos) } \\
\hline $20-39$ & 394 & $25,8(23,0 ; 28,7)$ \\
\hline $40-59$ & 560 & $36,6(34,0 ; 39,3)$ \\
\hline$\geq 60$ & 575 & $37,6(33,6 ; 41,8)$ \\
\hline \multicolumn{3}{|l|}{ Escolaridade * } \\
\hline Nenhuma/Ensino Fundamental & 604 & $44,4(38,9 ; 50,1)$ \\
\hline Ensino Médio & 567 & $41,7(38,5 ; 45,0)$ \\
\hline Ensino Superior & 188 & $13,8(10,4 ; 18,1)$ \\
\hline \multicolumn{3}{|l|}{ Cor da pele ** } \\
\hline Branca & 1.185 & $78,8(74,8 ; 82,4)$ \\
\hline Parda & 179 & $11,9(9,4 ; 15,0)$ \\
\hline Preta & 139 & $9,2(7,5 ; 11,4)$ \\
\hline \multicolumn{3}{|l|}{ Número de moradores no domicílio } \\
\hline $1-2$ & 747 & $48,8(44,3 ; 53,2)$ \\
\hline $3-4$ & 589 & $38,5(35,0 ; 42,1)$ \\
\hline$\geq 5$ & 196 & $12,8(10,4 ; 15,6)$ \\
\hline \multicolumn{3}{|c|}{ Presença de moradores menores de 18 anos no domicílio } \\
\hline Não & 992 & $64,0(59,0 ; 68,6)$ \\
\hline Sim & 558 & $36,0(31,4 ; 41,0)$ \\
\hline \multicolumn{3}{|l|}{ Número de trabalhadores por domicílio } \\
\hline Nenhum & 576 & $37,1(33,0 ; 41,5)$ \\
\hline Um & 551 & $35,6(32,8 ; 38,5)$ \\
\hline Dois ou mais & 423 & $27,3(24,2 ; 30,6)$ \\
\hline \multicolumn{3}{|l|}{ Distanciamento social } \\
\hline Muito pouco/Pouco & 197 & $12,8(10,2 ; 15,9)$ \\
\hline Mais ou menos & 357 & $23,2(20,1 ; 26,7)$ \\
\hline Praticamente isolado & 983 & $64,0(60,6 ; 67,2)$ \\
\hline \multicolumn{3}{|l|}{ Rotina de atividades } \\
\hline Fica em casa todo o tempo & 356 & $23,1(20,1 ; 26,3)$ \\
\hline Sai às vezes & 815 & $52,8(49,5 ; 56,1)$ \\
\hline Sai todos os dias & 372 & $24,1(21,3 ; 27,2)$ \\
\hline Total & 1.550 & 100,0 \\
\hline
\end{tabular}

* Variável com maior quantidade de perda de informações $(n=1.359)$.

** Amarelos e Indígenas não foram incluídos na estratificação desta variável, pois representavam apenas 0,53\% da amostra $(n=8)$. 
to social foram recomendadas no município. Por outro lado, um quarto dos indivíduos afirmou sair todos os dias de casa para trabalhar ou para outra atividade regular (Tabela 1).

A prevalência de insegurança alimentar geral foi de 29,4\% (IC95\%: 25,0; 34,4), apresentando uma diminuição porcentual entre o início de maio e o fim de junho, mas sem diferença significativa na análise de tendência (valor de $\mathrm{p}=0,069$ ). Em todas as rodadas do inquérito a insegurança alimentar tendeu a ser maior entre os adultos (20-39 e 40-59 anos), entre os entrevistados de menor escolaridade, de cor da pele preta ou parda, naqueles que residiam em domicílios com maior número de moradores e nos domicílios com moradores menores de 18 anos (Tabela 2). Análise de tendência temporal mostrou que, ao longo do tempo, a prevalência de insegurança alimentar reduziu de modo mais intenso entre os mais jovens (20-39 anos) e naqueles que residiam em domicílios com, no máximo, duas pessoas (Tabela 2).

A prevalência de insegurança alimentar não se mostrou estatisticamente associada ao número de trabalhadores por domicílio e às medidas de distanciamento social adotadas pelo município nas

\section{Tabela 2}

Tendência temporal da prevalência de insegurança alimentar de acordo com as variáveis sociodemográficas estudadas. Bagé, Rio Grande do Sul, Brasil $(n=1.550)$.

\begin{tabular}{|c|c|c|c|c|c|}
\hline $\begin{array}{l}\text { Características } \\
\text { sociodemográficas }\end{array}$ & $\begin{array}{l}\text { Rodada } 1 \\
\% \text { (IC95\%) }\end{array}$ & $\begin{array}{l}\text { Rodada } 2 \\
\% \text { (IC95\%) }\end{array}$ & $\begin{array}{l}\text { Rodada } 3 \\
\% \text { (IC95\%) }\end{array}$ & $\begin{array}{l}\text { Rodada } 4 \\
\% \text { (IC95\%) }\end{array}$ & $\begin{array}{c}\text { Variação } \\
\text { (valor de p) * }\end{array}$ \\
\hline Sexo & 0,179 & 0,273 & 0,899 & 0,594 & \\
\hline Masculino & $31,0(23,9 ; 39,3)$ & $25,0(17,7 ; 34,1)$ & $26,1(17,3 ; 37,4)$ & $25,0(17,2 ; 34,8)$ & $-1,7(0,191)$ \\
\hline Feminino & $38,1(30,9 ; 45,9)$ & $31,0(23,7 ; 39,4)$ & $26,8(20,3 ; 34,5)$ & $27,7(19,7 ; 37,5)$ & $-3,5(0,108)$ \\
\hline Idade (anos) & 0,004 & $<0,001$ & 0,006 & 0,003 & \\
\hline $20-39$ & $44,8(33,4 ; 56,8)$ & $37,9(26,7 ; 50,7)$ & $37,1(26,0 ; 49,7)$ & $28,4(19,2 ; 39,8)$ & $-5.0(0,017)$ \\
\hline $40-59$ & $40,4(30,5 ; 51,3)$ & $39,6(30,1 ; 49,9)$ & $29,6(21,5 ; 39,1)$ & $33,6(24,3 ; 44,3)$ & $-3,0(0,134)$ \\
\hline$\geq 60$ & $21,9(15,5 ; 30,0)$ & $15,9(10,0 ; 24,3)$ & $16,7(10,3 ; 25,8)$ & $16,0(10,2 ; 24,4)$ & $-1,7(0,204)$ \\
\hline Escolaridade & 0,626 & 0,149 & 0,036 & 0,037 & \\
\hline Nenhuma/Ensino & $36,7(30,5 ; 43,4)$ & $35,4(28,8 ; 42,7)$ & $34,9(25,5 ; 45,6)$ & $31,3(21,0 ; 43,8)$ & $-1,7(0,053)$ \\
\hline \multicolumn{6}{|l|}{ Fundamental } \\
\hline Ensino Médio & $39,1(29,6 ; 49,5)$ & $31,8(24,7 ; 39,7)$ & $25,3(17,9 ; 34,6)$ & $30,7(22,0 ; 41,1)$ & $-3,2(0.264)$ \\
\hline Ensino Superior & $31,6(19,4 ; 46,9)$ & $22,5(13,0 ; 36,2)$ & $11,1(3,6 ; 29,7)$ & $12,7(7,1 ; 21,9)$ & $-6,8(0,064)$ \\
\hline Cor da pele & 0,032 & 0,007 & $<0,001$ & 0,054 & \\
\hline Branca & $32,2(26,4 ; 38,7)$ & $25,3(19,9 ; 31,6)$ & $21,0(15,6 ; 27,9)$ & $23,5(16,8 ; 32,0)$ & $-3,0(0,179)$ \\
\hline Parda & $51,3(37,5 ; 64,9)$ & $44,2(27,7 ; 62,1)$ & $50,9(36,8 ; 64,9)$ & $38,1(26,5 ; 51,2)$ & $-3,3(0,189)$ \\
\hline Preta & $41,2(27,9 ; 55,9)$ & $47,4(32,2 ; 63,1)$ & $36,7(23,3 ; 52,5)$ & $32,4(18,4 ; 50,5)$ & $-3,7(0,195)$ \\
\hline $\begin{array}{l}\text { Número de moradores no } \\
\text { domicílio }\end{array}$ & 0,006 & 0,006 & 0,031 & 0,102 & \\
\hline $1-2$ & $25,3(18,8 ; 33,0)$ & $22,7(16,0 ; 31,0)$ & $22,6(15,4 ; 31,9)$ & $20,5(13,5 ; 30,0)$ & $-1,5(0,020)$ \\
\hline $3-4$ & $44,0(35,3 ; 53,1)$ & $33,8(26,1 ; 42,3)$ & $25,4(17,9 ; 34,6)$ & $32,6(22,7 ; 44,5)$ & $-4,3(0,269)$ \\
\hline$\geq 5$ & $42,6(28,3 ; 58,2)$ & $43,1(31,6 ; 55,5)$ & $43,9(30,5 ; 58,2)$ & $30,0(16,9 ; 47,5)$ & $-3,7(0,258)$ \\
\hline $\begin{array}{l}\text { Presença de menores de } 18 \\
\text { anos no domicílio }\end{array}$ & $<0,001$ & $<0,001$ & 0,010 & 0,007 & \\
\hline Não & $27,5(22,2 ; 33,5)$ & $22,0(15,8 ; 30,0)$ & $22,0(16,0 ; 29,5)$ & $20,5(14,0 ; 28,9)$ & $-2,1(0,095)$ \\
\hline Sim & $49,3(40,1 ; 58,5)$ & $42,8(34,3 ; 51,6)$ & $34,6(26,1 ; 44,2)$ & $35,9(25,9 ; 47,3)$ & $-4,8(0,067)$ \\
\hline Total & $35,2(29,9 ; 41,0)$ & $29,7(24,1 ; 35,9)$ & $26,4(20,5 ; 33,2)$ & $26,2(19,5 ; 34,1)$ & $-3,0(0.069)$ \\
\hline
\end{tabular}

IC95\%: intervalo de 95\% de confiança.

* Valor de p oriundo da análise de regressão linear para verificar a tendência do desfecho dentro de cada subgrupo analisado. 
quatro rodadas do inquérito epidemiológico em questão. No entanto, a análise de tendência temporal evidenciou que os domicílios que contavam com dois ou mais trabalhadores apresentaram queda mais acentuada na prevalência desse agravo. Além disso, a queda na prevalência de insegurança alimentar também foi maior entre os indivíduos que relataram conseguir cumprir muito pouco as medidas de distanciamento social e naqueles que afirmaram sair todos os dias de casa durante a pandemia (Tabela 3).

Análises complementares de desigualdade, baseadas em medidas absoluta e relativa da prevalência de insegurança alimentar de acordo com idade, escolaridade e número de moradores por domicílio evidenciaram que, durante os meses de maio e junho de 2020, a prevalência de insegurança alimentar esteve mais concentrada entre os indivíduos mais jovens, de menor escolaridade e que residiam em domicílios com maior número de moradores. Ademais, com exceção da escolaridade, para as demais variáveis os índices de desigualdades absoluta e relativa se mantiveram semelhantes nos quatro inquéritos epidemiológicos analisados, havendo leve recrudescimento da desigualdade na última rodada. Para a variável escolaridade, porém, a desigualdade relativa foi ampliada a partir da segunda rodada, já a desigualdade absoluta foi ampliada a partir da terceira rodada do estudo, indicando, neste caso, uma diferença de mais de 18 pontos percentuais entre os grupos extremos de desigualdade no último inquérito epidemiológico (Tabela 4).

\section{Tabela 3}

Tendência temporal da prevalência de insegurança alimentar de acordo com as variáveis relacionadas às medidas de distanciamento social. Bagé, Rio Grande do Sul, Brasil ( $\mathrm{n}=1.550)$.

\begin{tabular}{|c|c|c|c|c|c|}
\hline $\begin{array}{l}\text { Características de trabalho } \\
\text { e distanciamento social }\end{array}$ & $\begin{array}{l}\text { Rodada } 1 \\
\%(I C 95 \%)\end{array}$ & $\begin{array}{l}\text { Rodada } 2 \\
\%(I C 95 \%)\end{array}$ & $\begin{array}{l}\text { Rodada } 3 \\
\%(I C 95 \%)\end{array}$ & $\begin{array}{l}\text { Rodada } 4 \\
\%(I C 95 \%)\end{array}$ & $\begin{array}{c}\text { Variação } \\
\text { (valor de p) * }\end{array}$ \\
\hline $\begin{array}{l}\text { Número de trabalhadores por } \\
\text { domicílio }\end{array}$ & 0,307 & 0,082 & 0,709 & 0,283 & \\
\hline Nenhum & $29,9(22,0 ; 39,1)$ & $23,6(16,2 ; 33,1)$ & $25,3(17,1 ; 35,7)$ & $21,8(14,6 ; 31,3)$ & $-2,3(0,087)$ \\
\hline Um & $38,9(29,2 ; 49,5)$ & $33,6(27,1 ; 40,8)$ & $25,0(16,5 ; 36,0)$ & $29,6(21,5 ; 39,2)$ & $-3,7(0,167)$ \\
\hline Dois ou mais & $37,8(28,9 ; 47,6)$ & $33,0(24,4 ; 43,0)$ & $29,9(21,0 ; 40,6)$ & $26,7(17,1 ; 39,2)$ & $-3,6(0,005)$ \\
\hline Distanciamento social & 0,090 & 0,772 & 0,799 & 0,858 & \\
\hline Muito pouco/Pouco & $42,0(28,3 ; 57,0)$ & $31,8(18,7 ; 48,7)$ & $26,5(15,6 ; 41,3)$ & $24,6(15,9 ; 36,0)$ & $-5,8(0,048)$ \\
\hline Mais ou menos & $44,3(33,3 ; 55,8)$ & $32,2(21,5 ; 45,1)$ & $29,0(17,3 ; 44,4)$ & $28,0(17,3 ; 42,0)$ & $-5.2(0,099)$ \\
\hline $\begin{array}{l}\text { Bastante/Praticamente } \\
\text { isolado }\end{array}$ & $31,8(25,7 ; 38,7)$ & $28,4(22,6 ; 35,0)$ & $25,5(18,9 ; 33,4)$ & $25,6(17,9 ; 35,2)$ & $-2.2(0,062)$ \\
\hline Rotina de atividades & 0,474 & 0,381 & 0,657 & 0,978 & \\
\hline Fica em casa todo o tempo & $31,4(23,3 ; 40,8)$ & $32,1(22,5 ; 43,6)$ & $29,6(17,6 ; 45,3)$ & $25,6(15,4 ; 39,4)$ & $-2.0(0,117)$ \\
\hline Sai de vez em quando & $35,3(28,6 ; 42,5)$ & $27,0(20,9 ; 34,1)$ & $24,4(18,2 ; 31,9)$ & $25,8(18,4 ; 34,9)$ & $-3,1(0,180)$ \\
\hline Sai todos os dias & $39,8(27,9 ; 53,0)$ & $34,7(23,2 ; 48,3)$ & $28,1(18,2 ; 40,8)$ & $26,9(17,1 ; 39,4)$ & $-4,5(0,022)$ \\
\hline
\end{tabular}

IC95\%: intervalo de 95\% de confiança.

* Valor de p oriundo da análise de regressão linear para verificar a tendência do desfecho dentro de cada subgrupo analisado. 
Tabela 4

Desigualdades absoluta e relativa na insegurança alimentar de acordo com as variáveis sociodemográficas. Bagé, Rio Grande do Sul, Brasil ( $\mathrm{n}=1.550$ ).

\begin{tabular}{|c|c|c|c|c|}
\hline Características sociodemográficas & $\begin{array}{c}\text { Rodada } 1 \\
\text { (Valor de p) }\end{array}$ & $\begin{array}{c}\text { Rodada } 2 \\
\text { (Valor de } p \text { ) }\end{array}$ & $\begin{array}{c}\text { Rodada } 3 \\
\text { (Valor de } p \text { ) }\end{array}$ & $\begin{array}{c}\text { Rodada } 4 \\
\text { (Valor de p) }\end{array}$ \\
\hline \multicolumn{5}{|l|}{ SII } \\
\hline Idade (anos) & $-34,1(<0,001)$ & $-36,3(<0,001)$ & $-29,8(<0,001)$ & $-20,0(0,010)$ \\
\hline Escolaridade & $-3,4(0,731)$ & $-14,6(0,149)$ & $-27,3(0,002)$ & $-18,3(0,033)$ \\
\hline Número de moradores no domicílio & $31,4(<0,001)$ & $27,0(0,001)$ & $18,1(0,035)$ & $19,3(0,018)$ \\
\hline \multicolumn{5}{|l|}{ CIX } \\
\hline Idade (anos) & $-15,5(<0,001)$ & $-15,3(<0,001)$ & $-14,4(<0,001)$ & $-6,7(0,172)$ \\
\hline Escolaridade & $-5,6(0,150)$ & $-12,0(0,005)$ & $-14,6(0,002)$ & $-10,4(0,020)$ \\
\hline Número de moradores no domicílio & $11,6(0,003)$ & $14,5(0,001)$ & $14,5(0,003)$ & $12,6(0,009)$ \\
\hline
\end{tabular}

CIX: índice de concentração, que mede a desigualdade na prevalência de insegurança alimentar de acordo com idade, escolaridade e número de moradores no domicílio, utilizando abordagem similar à do coeficiente de Gini; SII: índice angular de desigualdade, obtido com base na regressão logística entre a prevalência de insegurança alimentar com idade, escolaridade e número de moradores no domicílio.

\section{Discussão}

Os resultados dos quatro inquéritos epidemiológicos sobre a COVID-19 realizados na cidade de Bagé evidenciaram que quase um terço dos domicílios estava enfrentando situação de insegurança alimentar nos meses de maio e junho de 2020. A prevalência de insegurança alimentar observada no presente estudo foi semelhante àquela encontrada em outro trabalho de base populacional realizado em Rio Grande, município também localizado no sul do Rio Grande do Sul e com características socioeconômicas semelhantes às de Bagé 30; no entanto, foi maior do que a prevalência observada na Região Sul do país, que era de 20,7\% em 2018 12. Por outro lado, considerando trabalhos realizados em outras regiões do país 10,14,31,32, a prevalência de insegurança alimentar neste estudo foi inferior, evidenciando a heterogeneidade da situação alimentar domiciliar no contexto brasileiro.

Embora as comparações apresentadas no parágrafo anterior sejam válidas para entender a insegurança alimentar no cenário nacional, no contexto da COVID-19 é interessante que a comparação seja feita com outros estudos que também tenham avaliado a insegurança alimentar durante a pandemia. Uma pesquisa realizada no México encontrou um aumento importante da insegurança alimentar durante a crise de saúde pública causada pelo SARS-CoV-2: de 61,1\% em abril para 75,1\% em junho de 2020 33, muito acima da observada no presente estudo.

Apesar das discrepâncias na forma como os dados foram coletados e como a insegurança alimentar foi avaliada em ambos os estudos, parte da diferença observada pode ser explicada pela maneira como os dois países estão enfrentando a pandemia. O México implementou um lockdown nacional entre 17 de março e 31 de maio, sendo que a maioria do país esteve em nível vermelho de risco, com somente os serviços essenciais funcionando mesmo após o governo ter flexibilizado o lockdown a partir de junho 33. Já o Brasil não chegou a adotar medida tão extrema em nível nacional, sendo que em alguns estados, como o Rio Grande do Sul, o retorno de diversas atividades aconteceu antes mesmo do pico de transmissão da doença. Especificamente no Município de Bagé, o retorno das atividades econômicas e comerciais começou a acontecer ainda em abril e maio, conforme os Decretos Municipais no 58, 59 e 68 de 2020 (http://bage.rs.gov.br).

Se por um lado a maneira como cada país conduziu o enfrentamento da pandemia pode ter sido crucial para a diferença no total de casos (32.154 casos por milhão de habitantes no Brasil vs. 9.385 casos por milhão de habitantes no México em dezembro de 2020), tal fato também pode explicar a diferença nas prevalências de insegurança alimentar em ambos os cenários, visto que medidas extremas de distanciamento social, embora achatem a curva de transmissão de epidemias como a causada pelo novo coronavírus, podem dificultar o acesso das famílias a uma alimentação adequada e saudável, 
principalmente daquelas em condições de maior vulnerabilidade social 34,35. Todavia, não podemos desconsiderar o fato de que o estudo mexicano foi feito em nível nacional, e a presente pesquisa foi realizada em apenas uma cidade do sul do Brasil, região que historicamente apresenta menor prevalência de insegurança alimentar quando comparada à média brasileira 10,12,36.

$O$ fato de a prevalência de insegurança alimentar não ter aumentado nos quatro inquéritos epidemiológicos analisados pode ser reflexo de dois fatores importantes. O primeiro deles é o auxílio emergencial, que começou a ser pago no mês de abril de 2020 como medida de proteção social para populações em vulnerabilidade 6, situação que pode impactar diretamente na segurança alimentar dos domicílios ${ }^{37}$. O segundo é o curto espaço de tempo entre o primeiro e o último inquéritos epidemiológicos, ocorridos no início de maio e no fim de junho de 2020, respectivamente. É de se esperar que em momentos de crise haja um período de latência até que a situação alimentar dos domicílios se deteriore de modo a alcançar situação de insegurança alimentar.

Desde a década de 1990, em especial a partir do início dos anos 2000, diversas políticas de alimentação e nutrição foram criadas com o objetivo de garantir o DHAA à população brasileira. Entre as diversas políticas criadas, podemos citar a Lei Orgânica de Segurança Alimentar e Nutricional 7, o Sistema Nacional de Segurança Alimentar e Nutricional e a Política Nacional de Segurança Alimentar e Nutricional 38. O esforço na constituição dessas políticas resultou em diminuição da prevalência de insegurança alimentar na primeira década dos anos 2000 8,9,10.

Porém, desde a crise político-econômica iniciada em 2015 no país observa-se um desmonte dessas políticas, culminando com a extinção do Conselho Nacional de Segurança Alimentar e Nutricional em 2019, órgão que, entre outras funções, exercia o controle social na formulação e execução de políticas e programas de segurança alimentar e nutricional. O desmonte de tais políticas associado à crise político-econômica do país culminaram em aumento da insegurança alimentar domiciliar, conforme atestado pelos dados da última POF 12. Presume-se que a situação de insegurança alimentar no país esteja sendo agravada pela pandemia de COVID-19, visto que esta crise repercute na oferta e no acesso à alimentação em quantidade suficiente e qualidade 20 , embora não tenhamos evidenciado isto no Município de Bagé.

Um agravamento da situação de insegurança alimentar domiciliar durante a pandemia de COVID-19 pode ter consequências importantes naquelas populações que já apresentavam risco maior de desfechos adversos. A insegurança alimentar pode diminuir a variedade e a qualidade da dieta 39,40 com consequente diminuição no consumo de frutas e hortaliças ${ }^{41}$, pode aumentar o risco de desnutrição no público infantil $42,43,44$ e a prevalência de obesidade em adultos 43 , principalmente em mulheres 45,46, aumentando consequentemente o risco de doenças crônicas não transmissíveis (DCNT) 43 . Toda essa cadeia de consequências associadas à insegurança alimentar tem impacto econômico e de saúde pública importante, podendo, inclusive, aumentar, a médio prazo, a taxa de mortalidade precoce das populações expostas a tal condição.

Desse modo, as políticas públicas a serem formuladas e desenvolvidas na pandemia e no pós-pandemia devem levar em consideração estratégias para diminuir a insegurança alimentar domiciliar, em especial a fome e, por conseguinte, minimizar as consequências da insegurança alimentar. Alpino et al. 36 destacaram ações importantes para mitigar os efeitos da pandemia no acesso universal à alimentação adequada e saudável, tais como instituição de uma renda básica emergencial, aporte financeiro para pequenas e médias empresas, repasse de recursos federais para ações de incremento à segurança alimentar e o desenvolvimento de medidas emergenciais na gestão do Programa Bolsa Família. Além disso, Ribeiro-Silva et al. 20 destacaram ainda o fortalecimento do Programa de Aquisição de Alimentos e a continuidade do Programa Nacional de Alimentação Escolar como medidas importantes para diminuir a situação de insegurança alimentar de famílias em vulnerabilidade social.

Como já amplamente descrito pela literatura, a insegurança alimentar está relacionada a diversos fatores sociodemográficos 10,14,46,47. Do mesmo modo, no presente estudo a insegurança alimentar esteve associada com escolaridade, cor da pele, idade, número de moradores por domicílio e presença de moradores menores de 18 anos de idade. Além disso, apesar da análise de tendência temporal ter mostrado que a prevalência de insegurança alimentar domiciliar se manteve estável entre o início de maio e o fim de junho, observou-se uma tendência de diminuição da insegurança alimentar mais acentuada naqueles domicílios com menor número de moradores e com dois trabalhadores ou mais. 
Tais resultados, principalmente o último, podem refletir uma recuperação da renda das famílias ocasionada pela manutenção do emprego ou também pelo auxílio emergencial, que começou a ser pago em abril de 2020 e que beneficiou trabalhadores informais e pessoas que tiveram a renda comprometida durante a pandemia. Um levantamento realizado pela PNAD COVID 48 mostra que, de fato, o auxílio emergencial teve impacto importante na situação de pobreza extrema observada no país. De acordo com a PNAD COVID, 3,3\% da população brasileira viviam em pobreza extrema em junho de 2020; menor nível nos últimos 40 anos 48. Em Bagé, especificamente, mais de 30 mil indivíduos foram beneficiados com o recebimento do auxílio emergencial até o momento, de acordo com o portal da transparência (http://www.portaldatransparencia.gov.br), fato que pode ter contribuído para a maior diminuição da insegurança alimentar entre os domicílios com, pelo menos, um trabalhador.

Outro aspecto importante a ser destacado neste estudo foi o fato de que, apesar da análise de tendência temporal não ter mostrado mudança na prevalência de insegurança alimentar de acordo com a escolaridade ao longo do tempo, quando realizadas as análises específicas de desigualdade observouse um leve aumento das medidas absoluta e relativa a partir da segunda rodada deste estudo, sugerindo uma concentração ainda maior da insegurança alimentar entre os menos escolarizados. Esse resultado pode ser consequência de alguma recuperação de renda e emprego entre os mais escolarizados, após o tombo recorde da economia no primeiro trimestre de 2020 1,2. De fato, a PNAD COVID mostrou que a taxa de desocupação é maior entre os indivíduos menos escolarizados 48, embora o desemprego tenha se mantido em patamares elevados em todos os subgrupos populacionais.

É importante salientar que o grupo de idosos esteve sobrerrepresentado neste estudo, visto que mais de 35\% dos entrevistados tinham 60 anos ou mais. De acordo com o último censo, a proporção de idosos em Bagé era de apenas 14,7\% em 2010. Como demonstrado por este e outros trabalhos 30,47, a prevalência de insegurança alimentar é menor na população idosa e tal fato pode ter contribuído para subestimar a prevalência deste agravo no presente estudo. A alta proporção de entrevistadas do sexo feminino (66\%) também merece destaque, pois aponta para a desigualdade de gênero na inserção no mercado de trabalho. Como as entrevistas ocorreram, em sua maioria, em dias de semana e em horário comercial, isso aumentou a probabilidade de terem sido encontradas mulheres e idosos nas residências, diminuindo a possibilidade de participação de homens nas entrevistas, haja vista que mesmo com as medidas de distanciamento social adotadas, diversas atividades econômicas e comerciais já haviam sido retomadas em Bagé na época em que o estudo aconteceu.

Por fim, destacam-se dois aspectos limitantes deste estudo relacionados ao questionário utilizado para mensurar a insegurança alimentar. $\mathrm{O}$ primeiro diz respeito à mudança do período recordatório da escala, dos usuais três meses para o início das medidas de distanciamento social. Apesar dessa mudança no período recordatório não ter sido previamente validada, acredita-se que não interferiu nos resultados, visto que o período recordatório englobado não foi maior que o da escala original, o que não ocasionaria risco de maior dificuldade de lembrar do que o existente para o período de referência original da escala. A outra limitação ligada à versão curta da EBIA está no fato de tal escala não detectar os diferentes níveis de intensidade da insegurança alimentar, não permitindo aos autores analisar a evolução temporal nas prevalências de insegurança alimentar moderada e grave, indicativas de um pior cenário na situação alimentar dos domicílios. No entanto, cabe considerar que mesmo sem ter sido devidamente validada e sem considerar os diferentes níveis de intensidade, essa escala é importante para o rastreamento das famílias em situação de insegurança alimentar 23 .

Como pontos positivos do presente estudo podemos considerar o fato de termos avaliado a insegurança alimentar durante a pandemia de COVID-19 com base em inquéritos seriados de base populacional, o que permitiu avaliarmos as tendências na insegurança alimentar durante os meses de maio e junho de 2020, baseando-se em um delineamento de baixo custo, de execução simples e com coleta de dados rápida e objetiva. Além disso, as análises complementares com a utilização de índices complexos de desigualdade para avaliar as desigualdades na insegurança alimentar ao longo do tempo também foi um ponto forte do trabalho por descrever a magnitude das diferenças observadas.

Em conclusão, este estudo mostrou uma forte associação da insegurança alimentar com aspectos sociodemográficos dos entrevistados, evidenciando maior concentração de tal agravo nos indivíduos mais jovens, menos escolarizados e que residiam em domicílios com cinco moradores ou mais. Os resultados aqui apresentados sugerem o potencial impacto da pandemia na situação alimentar dos domicílios e podem ser úteis no desenvolvimento de políticas e programas de combate à insegurança 
alimentar durante e após a pandemia. Tais políticas e programas são e serão extremamente importantes para dirimir os efeitos econômicos causados pela pandemia no DHAA da população de Bagé e do Brasil como um todo.

\section{Colaboradores}

L. P. Santos propôs a ideia e contribuiu com a concepção e desenho da pesquisa, análise e interpretação dos dados, edição e aprovação final da versão a ser publicada. A. A. Schäfer e F. O. Meller contribuiu com a análise e interpretação dos dados e com a escrita do manuscrito. J. Harter, B. P. Nunes, I. C. M. Silva e D. C. P. Pellegrini contribuíram com a concepção e desenho do estudo, interpretação dos resultados do artigo, redação e revisão crítica importante para o conteúdo intelectual do manuscrito. Todos os autores aprovaram a versão final a ser publicada.

\section{Informações adicionais}

ORCID: Leonardo Pozza dos Santos (0000-00023993-3786); Antônio Augusto Schäfer (0000-00028834-0434); Fernanda de Oliveira Meller (00000002-1174-4721); Jenifer Harter (0000-00029130-4290); Bruno Pereira Nunes (0000-00024496-4122); Inácio Crochemore Mohnsam da Silva (0000-0001-5390-8360); Debora da Cruz Payão Pellegrini (0000-0002-4285-5643).

\section{Agradecimentos}

Agradecemos aos participantes da pesquisa e, pela pactuação na proposição do estudo e mobilização da equipe de coleta de dados, aos trabalhadores e trabalhadoras da Secretaria de Saúde e Atenção à Pessoa com Deficiência de Bagé, representados aqui nominalmente por Sheila Lucas de Silveira Tavares, da Coordenadoria de Vigilância Epidemiológica.

\section{Referências}

1. Coordenação de Contas Nacionais, Instituto Brasileiro de Geografia e Estatística. Contas nacionais trimestrais: 1o trimestre de 2020. Rio de Janeiro: Instituto Brasileiro de Geografia e Estatística; 2020.

2. Instituto Brasileiro de Geografia e Estatística. Indicadores IBGE: Pesquisa Nacional por Amostra de Domicílios contínua. Rio de Janeiro: Instituto Brasileiro de Geografia e Estatísticas; 2020.

3. Ministério do Desenvolvimento Social. O direito humano à alimentação adequada e o sistema nacional de segurança alimentar e nutricional. Brasília: Ação Brasileira pela Nutrição e Direitos Humanos; 2013.

4. Watts J. Brazil's health system woes worsen in economic crisis. Lancet 2016; 387:1603-4.

5. The Lancet Global Health. Food insecurity will be the sting in the tail of COVID-19. Lancet Glob Health 2020; 8:e737.

6. Brasil. Lei no 13.982, de 2 de abril de 2020. Altera a Lei no 8.742, de 7 de dezembro de 1993, para dispor sobre parâmetros adicionais de caracterização da situação de vulnerabilidade social para fins de elegibilidade ao benefício de prestação continuada (BPC), e estabelece medidas excepcionais de proteção social a serem adotadas durante o período de enfrentamento da emergência de saúde pública de importância internacional decorrente do coronavírus (Covid-19) responsável pelo surto de 2019, a que se refere a Lei no 13.979 , de 6 de fevereiro de 2020. Diário Oficial da União 2020; 2 abr.

7. Brasil. Lei no 11.346 , de 15 de setembro de 2006. Cria o Sistema Nacional de Segurança Alimentar e Nutricional com vistas a assegurar o direito humano à alimentação adequada e dá outras providências. Diário Oficial da União 2006; 18 set.

8. Instituto Brasileiro de Geografia e Estatística. Segurança alimentar, 2004: Pesquisa Nacional por Amostra de Domicílios. Rio de Janeiro: Instituto Brasileiro de Geografia e Estatística; 2006.

9. Instituto Brasileiro de Geografia e Estatística. Pesquisa Nacional por Amostra de Domicílio: segurança alimentar 2013. Rio de Janeiro: Instituto Brasileiro de Geografia e Estatística; 2014. 
10. Santos TG, Silveira JAC, Longo-Silva G, Ramires EKNM, Menezes RCE. Tendência e fatores associados à insegurança alimentar no Brasil: Pesquisa Nacional por Amostra de Domicílios 2004, 2009 e 2013. Cad Saúde Pública 2018; 34:e00066917.

11. Sousa LRM, Segall-Corrêa AM, Ville AS, Melgar-Quiñonez H. Food security status in times of financial and political crisis in Brazil. Cad Saúde Pública 2019; 35:e00084118.

12. Coordenação de Trabalho e Rendimento, Instituto Brasileiro de Geografia e Estatística. Pesquisa de orçamentos familiares 2017-2018: análise da segurança alimentar no Brasil. Rio de Janeiro: Instituto Brasileiro de Geografia e Estatística; 2020.

13. Committee on World Food Security. Interim issues paper on the impact of Covid-19 on food security and nutrition (FSN). Rome: Food and Agriculture Organization of the United Nations; 2020.

14. Trivellato PT, Morais DC, Lopes SO, Miguel ES, Franceschini SCC, Priore SE. Insegurança alimentar e nutricional em famílias do meio rural brasileiro: revisão sistemática. Ciênc Saúde Colet 2019; 24:865-74.

15. Akter S, Basher SA. The impacts of food price and income shocks on household food security and economic well-being: evidence from rural Bangladesh. Glob Environ Change 2014; 25:150-62.

16. Vilar-Compte M, Sandoval-Olascoaga S, Bernal-Stuart A, Shimoga S, Vargas-Bustamante A. The impact of the 2008 financial crisis on food security and food expenditures in Mexico: a disproportionate effect on the vulnerable. Public Health Nutr 2015; 18:2934-42.

17. Food Security Information Network. 2020 Global report on food crisis: joint analysis for better decisions. Rome: Food Security Information Network; 2020.

18. Oxfam Brasil. País estagnado: um retrato das desigualdades brasileiras. São Paulo: Oxfam Brasil; 2018.

19. Pitombeira DF, Oliveira LC. Pobreza e desigualdades sociais: tensões entre direitos, austeridade e suas implicações na atenção primária. Ciênc Saúde Colet 2020; 25:1699-708.

20. Ribeiro-Silva RC, Pereira M, Campello T, Aragão É, Guimarães JMM, Ferreira AJ, et al. Implicações da pandemia COVID-19 para a segurança alimentar e nutricional no Brasil. Ciênc Saúde Colet 2020; 25:3421-30.

21. Prefeitura Municipal de Bagé. Decreto $\mathrm{Mu}-$ nicipal no 050, de 19 de março de 2020. Declara estado de calamidade pública em toda a extensão do Município de Bagé, do Estado do Rio Grande do Sul para fins de prevenção e de enfrentamento à epidemia causada pelo COVID-19 (novo coronavírus), e dá outras providências. Diário Oficial Municipal 2020; 20 mar.
22. Hallal PC, Horta BL, Barros AJD, Dellagostin OA, Hartwig FP, Pellanda LC, et al. Evolução da prevalência de infecção por COVID-19 no Rio Grande do Sul, Brasil: inquéritos sorológicos seriados. Ciênc Saúde Colet 2020; 25 Suppl 1:2395-401.

23. Santos LP, Lindemann IL, Motta JVS, Mintem G, Bender E, Gigante DP. Proposal of a shortform version of the Brazilian Food Insecurity Scale. Rev Saúde Pública 2014; 48:783-9.

24. Prais S, Winsten C. Trend estimators and serial correlation. Chicago: Cowles Foundation; 1954. (Discussion Paper, 383).

25. Breusch TS. Testing for autocorrelation in dynamic linear models. Australian Economic Paper 1978; 17:334-55

26. Godfrey LG. Testing against general autoregressive and moving average error models when the regressors include lagged dependent variables. Econometrica 1978; 46:1293.

27. Barros AJD, Victora CG. Measuring coverage in $\mathrm{MNCH}$ : determining and interpreting inequalities in coverage of maternal, newborn, and child health interventions. PLoS Med 2013; 10:e1001390.

28. Silva ICM, Restarepo-Mendez MC, Costa JC, Ewerling F, Hellwig F, Ferreira LZ, et al. Mensuração de desigualdades sociais em saúde: conceitos e abordagens metodológicas no contexto brasileiro. Epidemiol Serv Saúde 2018; 27:e000100017.

29. Schneider MC, Castillo-Salgado C, Bacallao J, Loyola E, Mujica OJ, Vidaurre M, et al. Métodos de mensuração das desigualdades em saúde. Rev Panam Salud Pública 2002; 17:1-16.

30. Dias MS, Dumith SC, Vaz JS, Susin LRO. Insegurança alimentar em um município do extremo sul do Rio Grande do Sul, 2016: um estudo de base populacional. Epidemiol Serv Saúde 2020; 2020; 29:e2019204.

31. Bezerra TA, Olinda RA, Pedraza DF. Insegurança alimentar no Brasil segundo diferentes cenários sociodemográficos. Ciênc Saúde Colet 2017; 22:637-51.

32. Santos EES, Oliveira MM, Bernardino IM, Pedraza DF. Insegurança alimentar e nutricional de famílias usuárias da Estratégia Saúde da Família em dois municípios paraibanos, Brasil. Ciênc Saúde Colet 2020; 25:1607-17.

33. Gaitan-Rossi P, Vilar-Compte M, Teruel G, Perez-Escamilla R. Measurement lessons of a repeated cross-sectional household food insecurity survey during the COVID-19 pandemic in Mexico. medRxiv 2020; 6 ago. http://me drxiv.org/lookup/doi/10.1101/2020.08.04.20 167650.

34. Food and Agriculture Organization of the United Nations. Urban food systems and COVID-19: the role of cities and local governments in responding to the emergency. Rome: Food and Agriculture Organization of the United Nations; 2020. 
35. Huizar MI, Arena R, Laddu DR. The global food syndemic: the impact of food insecurity, Malnutrition and obesity on the healthspan amid the COVID-19 pandemic. Prog Cardiovasc Dis 2020; [Epub ahead of print].

36. Poblacion AP, Marín-León L, Segall-Corrêa AM, Silveira JA, Taddei JAAC. Insegurança alimentar em domicílios brasileiros com crianças menores de cinco anos. Cad Saúde Pública 2014; 30:1067-78.

37. Alpino TMA, Santos CRB, Barros DC, Freitas CM. COVID-19 e (in)segurança alimentar e nutricional: ações do Governo Federal brasileiro na pandemia frente aos desmontes orçamentários e institucionais. Cad Saúde Pública 2020; 36:e00161320.

38. Brasil. Decreto no 7272 , de 25 de agosto de 2010. Regulamenta a Lei no 11.346 , de 15 de setembro de 2006, que cria o Sistema Nacional de Segurança Alimentar e Nutricional SISAN - com vistas a assegurar o direito humano à alimentação adequada, institui a Política Nacional de Segurança Alimentar e Nutricional - PNSAN, estabelece os parâmetros para a elaboração do Plano Nacional de Segurança Alimentar e Nutricional, e dá outras providências. Diário Oficial da União 2010; 26 ago.

39. Kirkpatrick SI, Tarasuk V. Food insecurity is associated with nutrient inadequacies among Canadian adults and adolescents. J Nutr 2008; 138:604-12.

40. Leung CW, Epel ES, Ritchie LD, Crawford PB, Laraia BA. Food insecurity is inversely associated with diet quality of lower-income adults. J Acad Nutr Diet 2014; 114:1943-53.e2.
41. Panigassi G, Segall-Corrêa AM, Marin-León L, Pérez-Escamilla R, Maranha LK, Sampaio MFA. Insegurança alimentar intrafamiliar e perfil de consumo de alimentos. Rev Nutr 2008; 21 Suppl:135s-44s.

42. Santos LP, Gigante DP. Relationship between food insecurity and nutritional status of Brazilian children under the age of five. Rev Bras Epidemiol 2013; 16:984-94.

43. Pérez-Escamilla R, Cunningham K, Moran VH. COVID-19 and maternal and child food and nutrition insecurity: a complex syndemic. Matern Child Nutr 2020; 16:e13036.

44. Lopes AF, Frota MTBA, Leone C, Szarfarc SC. Perfil nutricional de crianças no Estado do Maranhão. Rev Bras Epidemiol 2019; 22:e190008.

45. Wilde PE, Peterman JN. Individual weight change is associated with household food security status. J Nutr 2006; 136:1395-400.

46. Morais DC, Dutra LV, Franceschini SCC, Priore SE. Insegurança alimentar e indicadores antropométricos, dietéticos e sociais em estudos brasileiros: uma revisão sistemática. Ciênc Saúde Colet 2014; 19:1475-88.

47. Maas NM, Mendoza-Sassi RA, Meucci RD, Cesar JA. Insegurança alimentar em famílias de área rural do extremo sul do Brasil. Ciênc Saúde Colet 2020; 25:2605-14.

48. Instituto Brasileiro de Geografia e Estatística. Pesquisa Nacional por Amostras de Domicílios: PNAD Covid. https://www.ibge.gov.br/ estatisticas/sociais/trabalho/27946-divulga cao-semanal-pnadcovid $1 . h t m l ?=\& \mathrm{t}=\mathrm{o}$-que-e (acessado em Ago/2020). 


\section{Abstract}

The objective was to analyze trends and inequalities in the prevalence of food insecurity during the COVID-19 pandemic according to sociodemographic factors and social distancing measures. We analyzed data from four serial epidemiological surveys on COVID-19 in May and June 2020, with adults and elderly living in Bagé, Rio Grande do Sul State, Brazil. Food insecurity was assessed with the short version of the Brazilian Food Insecurity Scale (EBIA), with the recall period adapted to the beginning of the social distancing period in the city. Sociodemographic characteristics and the adoption of social distancing measures were analyzed, and their associations with food insecurity were assessed with chi-square test. The temporal trend in food insecurity according to these characteristics was assessed via linear regression. Inequalities in food insecurity were assessed with the angular inequality index and concentration index. Of the 1,550 individuals studied, 29.4\% (95\%CI: 25.0; 34.4) presented food insecurity. Analysis of inequality showed higher concentration of food insecurity among the younger and less educated and those living with five or more residents in the same household. Over the course of the four surveys, prevalence of food insecurity decreased most sharply among the younger, those living in households with up to two residents, and those with two or more workers. There was a strong association between food insecurity and sociodemographic factors, which may indicate the pandemic's potential economic impact on households' food situation.

Food and Nutrition Security; COVID-19; Nutritional Epidemiology

\section{Resumen}

El objetivo fue analizar tendencias y desigualdades en la prevalencia de inseguridad alimentaria durante la pandemia de COVID-19, de acuerdo con factores sociodemográficos, así como con las medidas de distanciamiento social. Se analizaron datos de cuatro encuestas epidemiológicas seriadas sobre la COVID-19, desarrolladas entre mayo y junio de 2020, con adultos y ancianos residentes en la ciudad de Bagé, Rio Grande do Sul, Brasil. La inseguridad alimentaria se evaluó a través de la versión corta de la Escala Brasileña de Inseguridad Alimentaria (EBIA), con un periodo recordatorio, adaptado al inicio de las medidas de distanciamiento social en el municipio. Fueron analizadas características sociodemográficas y la adopción de medidas de distanciamiento social, así como sus asociaciones con la inseguridad alimentaria, utilizándose un test de chi-cuadrado. Se evaluó la tendencia temporal de la inseguridad alimentaria de acuerdo con tales características, utilizándose la regresión lineal. Se evaluaron desigualdades en la inseguridad alimentaria, mediante el indice angular de desigualdad y el índice de concentración. De los 1.550 individuos estudiados, un 29,4\% (IC95\%: $25,0 ; 34,4)$ presentaron inseguridad alimentaria. El análisis de desigualdad mostró una mayor concentración de inseguridad alimentaria entre los más jóvenes, los menos escolarizados, y quienes residian en domicilios con cinco residentes o más. A lo largo de las cuatro encuestas, la prevalencia de inseguridad alimentaria se redujo más acentuadamente entre los más jóvenes, en quienes residían en domicilios con hasta dos residentes y con dos o más trabajadores. Se evidenció una fuerte asociación de la inseguridad alimentaria con aspectos sociodemográficos de los entrevistados, lo que puede indicar el potencial impacto económico de la pandemia en la situación alimentaria de los domicilios.

Seguridad Alimentaria y Nutricional; COVID-19; Epidemiología Nutricional
Recebido em 16/Set/2020

Versão final reapresentada em 08/Jan/2021

Aprovado em 06/Fev/2021 